\title{
Influence of loopback interference channel in energy harvesting full-duplex relaying network over block rayleigh fading channel: performance analysis
}

\author{
Phu Tran Tin", Van-Duc Phan², Le Anh Vu ${ }^{3}$ \\ ${ }^{1}$ Faculty of Electronics Technology, Industrial University of Ho Chi Minh City, Ho Chi Minh City, Vietnam \\ ${ }^{2}$ Faculty of Automobile Technology, Van Lang University, Ho Chi Minh City, Vietnam \\ ${ }^{3}$ Optoelectronics Research Group, Faculty of Electrical and Electronics Engineering, Ton Duc Thang University, \\ Ho Chi Minh City, Vietnam
}

\begin{abstract}
Article Info
Article history:

Received Dec 10, 2020

Revised Jun 16, 2021

Accepted Jun 26, 2021

Keywords:

Cooperative network

ABSTRACT

The main idea of this paper is to investigate the system performance (SP) of energy harvesting FD relaying network over block rayleigh fading channel under the influence of the loopback interference channel. In the first stage, we proposed the system model and analyzed the energy harvesting and the information transmission phases. Furthermore, the mathematical form for the outage probability (OP) is analyzed and derived in two kinds of loopback interference: residual self-interference is modeled as AWGN và residual selfinterference is still a random variable. All the mathematical, analytical expressions are verified using the Monte Carlo simulation.
\end{abstract}

Energy harvesting

Loopback interference

Monte carlo simulation

Outage probability

This is an open access article under the $\underline{C C B Y-S A}$ license.

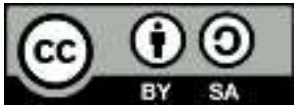

Corresponding Author:

Van-Duc Pha

Faculty of Automobile Technology

Van Lang University

Ho Chi Minh City, Vietnam

Email: duc.pv@vlu.edu.vn

\section{INTRODUCTION}

In the last recent years, so many papers focus on the energy harvesting $(\mathrm{RH})$ communication network [1]-[5]. The authors in [6]-[8] proposed and investigated the cooperative wireless networks by a MIMO relay system, the EH process, and how to improve this EH process in them. Furthermore, the multiuser and multi-hop systems and the energy and information transferring at the same time in the communication network is presented in [9]-[11]. The physical layer security problem in the EH communication network and its efficiency is investigated in [12]-[15]. From this literature, we can see that the investigation of the system performance of the EH communication network is necessary to study.

The main idea of this paper is to investigate the system performance (SP) of energy harvesting FD relaying network over block rayleigh fading channel under the influence of the loopback interference channel. In the first stage, we proposed the system model and analyzed the energy harvesting and the information transmission phases. Furthermore, the mathematical form for the outage probability (OP) is analyzed and derived in two kinds of loopback interference: residual self-interference is modeled as AWGN và residual self-interference is still a random variable. All the mathematical, analytical expressions are verified using the Monte Carlo simulation. 


\section{SYSTEM MODEL}

In this paper, the system model is illustrated in Figure 1, and the PSP is drawn in Figure 2. The Energy Harvesting and Information transmission phases can be formulated as (1)-(9) [16]. The received signal at the relay node can be given by (1),

$$
y_{R}=\sqrt{\rho} h_{S R} x_{s}+h_{R R} x_{R}+n_{R}
$$

where $h_{R R}$ : residual self-interference channel.

The harvested energy at the relay can be calculated by (2),

$$
E_{R}=\eta(1-\rho) T P_{s}\left|h_{S R}\right|^{2}
$$

from (2), we have,

$$
P_{R}=\frac{E_{R}}{T}=\eta(1-\rho) P_{s}\left|h_{S R}\right|^{2}=\kappa P_{s}\left|h_{S R}\right|^{2}
$$

next, the received signal at the destination can be expressed by (4),

$$
y_{D}=h_{R D} x_{R}+n_{D}
$$

the amplification factor $\beta$ will be determined as (5),

$$
\beta=\frac{x_{R}}{y_{R}}=\sqrt{\frac{P_{R}}{\left|h_{S R}\right|^{2} P_{s}+\left|h_{R R}\right|^{2} P_{R}+N_{0}}}
$$

from (4) and combining with (5), we can obtain,

$$
y_{D}=h_{R D} \beta y_{R}+n_{D}=h_{R D} \beta\left[\sqrt{\rho} h_{S R} x_{s}+h_{R R} x_{R}+n_{R}\right]+n_{D}=\underbrace{\sqrt{\rho} h_{R D} h_{S R} x_{s} \beta}_{\text {signal }}+\underbrace{h_{R D} \beta h_{R R} x_{R}}_{\text {intarferenet }}+\underbrace{h_{R D} \beta n_{R}+n_{D}}_{\text {noise }}
$$

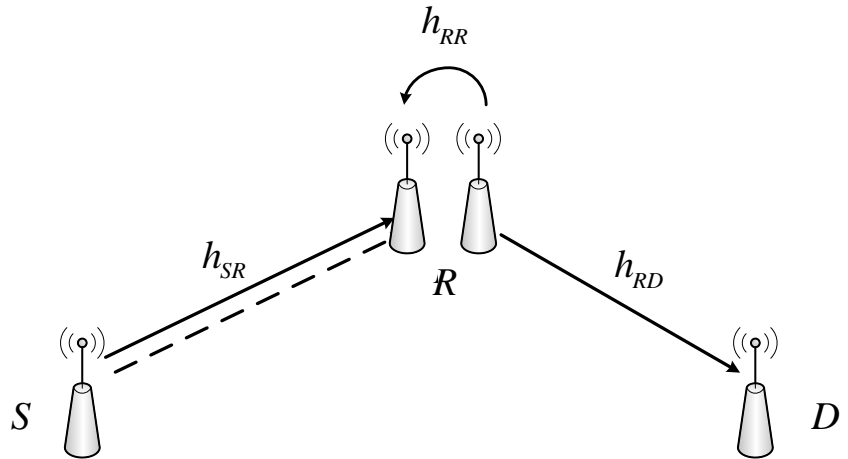

\section{$\longrightarrow \quad$ EH Phase $\quad-\rightarrow \quad$ IT Phase \\ $\longrightarrow \quad$ EH Phase $\quad-\rightarrow \quad$ IT Phase}

Figure 1. The proposed system

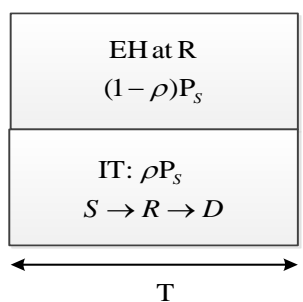

Figure 2. The power splitting phase

after doing some algebra, the SINR can be claimed as (9), 


$$
\begin{aligned}
\operatorname{SINR}_{A F} & =\frac{\mathrm{E}\left\{\mid \text { signal }\left.\right|^{2}\right\}}{\mathrm{E}\left\{\mid \text { inteference }\left.\right|^{2}\right\}+\mathrm{E}\left\{\mid \text { noise }\left.\right|^{2}\right\}}=\frac{\frac{\rho P_{s}\left|h_{S R}\right|^{2}\left|h_{R D}\right|^{2}}{\left|h_{R R}\right|^{2}}}{\frac{N_{0} P_{s}\left|h_{S R}\right|^{2}}{P_{R}\left|h_{R R}\right|^{2}}+P_{R}\left|h_{R D}\right|^{2}+\rho N_{0}} \\
& =\frac{\rho \kappa \Phi\left|h_{S R}\right|^{2}\left|h_{R D}\right|^{2}}{\kappa^{2} \Phi\left|h_{S R}\right|^{2}\left|h_{R D}\right|^{2}\left|h_{R R}\right|^{2}+\kappa\left|h_{R R}\right|^{2}+\rho}
\end{aligned}
$$

where $\Phi=\frac{P_{s}}{N_{0}}$ and $\kappa=\eta(1-\rho)$

\section{THE SYSTEM PERFORMANCE}

Case 1: residual self-interference is still a random variable (RV), the $\mathrm{OP}$ of the system at the source destination can be defined as (10),

$$
O P_{\text {casel }}=\operatorname{Pr}\left(\operatorname{SINR}_{A F}<\gamma_{t h}\right)=\operatorname{Pr}\left(\frac{\rho \kappa \Phi\left|h_{S R}\right|^{2}\left|h_{R D}\right|^{2}}{\kappa^{2} \Phi\left|h_{S R}\right|^{2}\left|h_{R D}\right|^{2}\left|h_{R R}\right|^{2}+\kappa\left|h_{R R}\right|^{2}+\rho}<\gamma_{t h}\right)
$$

where $\gamma_{t h}=2^{R}-1$ is the threshold of the system and $\mathrm{R}$ is the target rate.

Let us denote $X=\left|h_{S R}\right|^{2}\left|h_{R D}\right|^{2}$ and $Y=\left|h_{R R}\right|^{2}$, the (10) can be rewritten by (11),

$$
\begin{aligned}
O P_{\text {case } 1}= & \operatorname{Pr}\left(\frac{\rho \kappa \Phi X}{\kappa^{2} \Phi X Y+\kappa Y+\rho}<\gamma_{t h}\right) \\
& =\left\{\begin{array}{l}
\operatorname{Pr}\left(X<\frac{\gamma_{t h}(\kappa Y+\rho)}{\rho \kappa \Phi-\kappa^{2} \Phi Y \gamma_{t h}}\right), Y<\frac{\rho}{\kappa \gamma_{t h}} \\
1 \quad, Y \geq \frac{\rho}{\kappa \gamma_{t h}} \\
= \\
=\int_{0}^{\frac{\rho}{\kappa \gamma_{t h}}} F_{X}\left(\frac{\gamma_{t h}(\kappa y+\rho)}{\rho \kappa \Phi-\kappa^{2} \Phi y \gamma_{t h}}\right) \times f_{Y}(y) d y+\int_{\frac{\rho}{\kappa \gamma_{t h}}}^{\infty} f_{Y}(y) d y
\end{array}\right.
\end{aligned}
$$

in order to find the probability in (11), we have to calculate the cumulative distribution function (CDF) of X. So, the CDF of X can be found as (12),

$$
\begin{aligned}
F_{X}(x) & =\operatorname{Pr}(X<x)=\operatorname{Pr}\left(\left|h_{S R}\right|^{2}\left|h_{R D}\right|^{2}<x\right)=\int_{0}^{\infty} F_{\left|h_{S R}\right|^{2}}\left(\left.\frac{x}{y}|| h_{R D}\right|^{2}=y\right) \times f_{\left|h_{R D}\right|^{2}}(y) d y \\
& =1-\int_{0}^{\infty} \lambda_{R D} \exp \left(-\frac{\lambda_{S R} x}{y}-\lambda_{R D} y\right) d y
\end{aligned}
$$

where $\lambda_{S R}, \lambda_{R D}$ are the mean of RVs $\left|h_{S R}\right|^{2}$ and $\left|h_{R D}\right|^{2}$, respectively.

By applying the (12) [3.324,1] of [17], $F_{X}(x)$ can be reformulated by,

$$
F_{X}(x)=1-2 \sqrt{\lambda_{S R} \lambda_{R D} x} \times K_{1}\left(2 \sqrt{\lambda_{S R} \lambda_{R D} x}\right)
$$

where $K_{v}(\bullet)$ is the modified Bessel function of the second kind and v-th order.

Applying the (13), the $O P_{\text {casel }}$ can be rewritten by (14), 


$$
O P_{\text {casel }}=1-2 \lambda_{R R} \int_{0}^{\frac{\rho}{\kappa \gamma / h}} \exp \left(-\lambda_{R R} y\right) \times \sqrt{\frac{\lambda_{S R} \lambda_{R D} \gamma_{t h}(\kappa y+\rho)}{\rho \kappa \Phi-\kappa^{2} \Phi y \gamma_{t h}}} \times K_{1}\left(2 \sqrt{\frac{\lambda_{S R} \lambda_{R D} \gamma_{t h}(\kappa y+\rho)}{\rho \kappa \Phi-\kappa^{2} \Phi y \gamma_{t h}}}\right) d y
$$

Case 2: residual self-interference is modeled as AWGN with zero mean and variance $\Omega_{R R}$ [1]. In this case, from (9), the SINR can be rewritten by,

$$
\overline{S I N R_{A F}}=\frac{\rho \kappa \Phi\left|h_{S R}\right|^{2}\left|h_{R D}\right|^{2}}{\kappa^{2} \Phi\left|h_{S R}\right|^{2}\left|h_{R D}\right|^{2} \Omega_{R R}+\kappa \Omega_{R R}+\rho}
$$

the $\mathrm{OP}$ in case 2 can be calculated as,

$$
\begin{aligned}
O P_{\text {case } 2} & =\operatorname{Pr}\left(\overline{S I N R_{A F}}<\gamma_{t h}\right)=\operatorname{Pr}\left(\frac{\rho \kappa \Phi X}{\kappa^{2} \Phi X \Omega_{R R}+\kappa \Omega_{R R}+\rho}<\gamma_{t h}\right) \\
& =\operatorname{Pr}\left(X\left[\rho \kappa \Phi-\kappa^{2} \Phi \Omega_{R R} \gamma_{t h}\right]<\gamma_{t h}\left[\kappa \Omega_{R R}+\rho\right]\right)
\end{aligned}
$$

so that probability in (16) is available. It means that our system can be operated, we must estimate $\Omega_{R R}$, so that satisfy the condition: $\Omega_{R R}<\frac{\rho}{\kappa \gamma_{t h}}$ [18-21].

Hence, the $O P_{\text {case } 2}$ can be claimed by applying result from (13),

$$
\begin{aligned}
O P_{\text {case } 2} & =\operatorname{Pr}\left(X<\frac{\gamma_{t h}\left[\kappa \Omega_{R R}+\rho\right]}{\rho \kappa \Phi-\kappa^{2} \Phi \Omega_{R R} \gamma_{t h}}\right) \\
& =1-2 \sqrt{\frac{\lambda_{S R} \lambda_{R D} \gamma_{t h}\left(\kappa \Omega_{R R}+\rho\right)}{\rho \kappa \Phi-\kappa^{2} \Phi \Omega_{R R} \gamma_{t h}}} \times K_{1}\left(2 \sqrt{\frac{\lambda_{S R} \lambda_{R D} \gamma_{t h}\left(\kappa \Omega_{R R}+\rho\right)}{\rho \kappa \Phi-\kappa^{2} \Phi \Omega_{R R} \gamma_{t h}}}\right)
\end{aligned}
$$

\section{NUMERICAL RESULTS AND DISCUSSION}

As in [22]-[25], we use the Monte Carlo simulation for convincing the correctness of the analytical section. The influence of $\psi$ and $\rho$ on the system performance in terms of OP is shown in Figure 3 and Figure 4, respectively. In Figure 3, the main system parameters are set as $\mathrm{R}=1 \mathrm{bps} / \mathrm{Hz}, \rho=0.85, \eta=1$. As a result, the system OP has a massive fall with increasing of $\Phi$ from $-5 \mathrm{~dB}$ to $20 \mathrm{~dB}$ in both cases. Similarly, the system OP has a massive increase with rising of $\rho$ from 0 to 1 . In Figure 4, we set the primary system parameters as $R=1$ bps, $\Phi=5 \mathrm{~dB}, \eta=1,0.5$, and $\Omega_{\mathrm{RR}}=0$ and $0.05 \mathrm{~dB}$ for both cases. In Figure 4 and Figure 5, the simulation and analytical value are the same for convincing the analytical section.

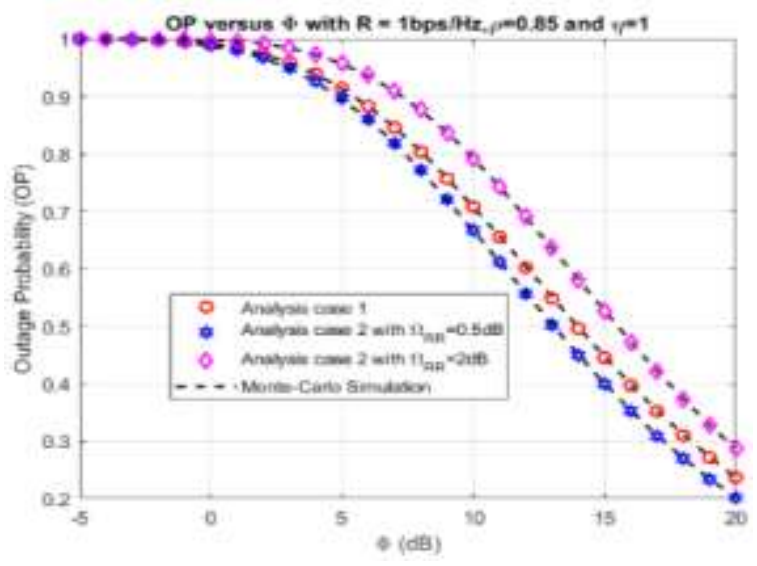

Figure 3. OP versus $\psi$

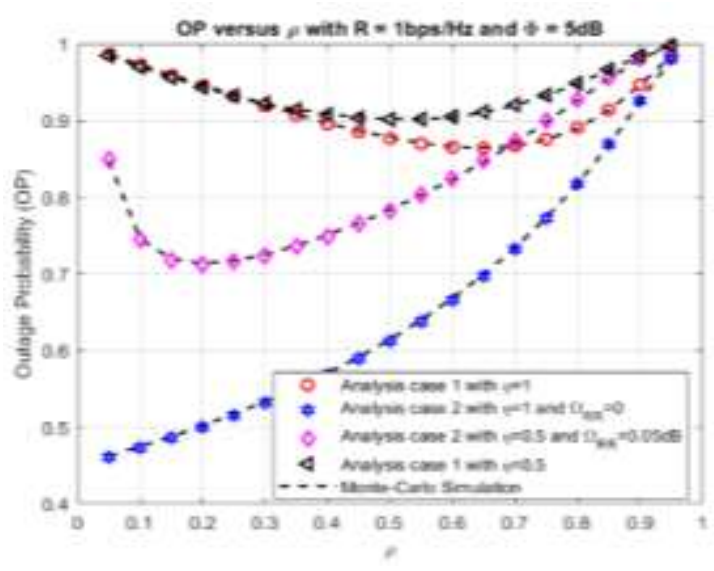

Figure 4. OP versus $\rho$ 
Figure 4 shows the OP versus $\rho$. Furthermore, the system OP as the function of $\eta$, while $\eta$ varies from 0 to 1 is illustrated in Figure 5. In this figure, the system parameters are set as $R=1 \mathrm{bps}, \Phi=5 \mathrm{~dB}, \rho=0.25$, 0.85 , and $\Omega_{\mathrm{RR}}=0.25$ and $2.5 \mathrm{~dB}$ for both cases. The system OP falls with the rising of $\eta$. On the other hand, the system $\mathrm{OP}$ as the function of $\mathrm{R}$ when $\mathrm{R}$ increases from 0 to $6 \mathrm{bps} / \mathrm{Hz}$ is shown in Figure 6 with the main system parameters as $\rho=0.5, \Phi=7 \mathrm{~dB}, \eta=1$ and $\Omega_{R R}=0 \mathrm{~dB}$. As shown in Figure 6 , the system OP has a rise with R. In both Figure 5 and Figure 6 , the simulation curves overlap the analytical curves to verify the mathematical section's correctness.

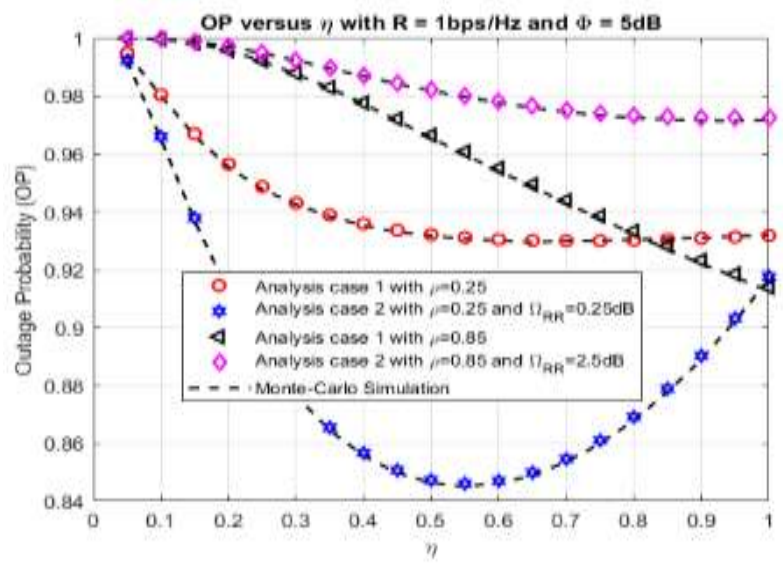

Figure 5. OP versus $\eta$

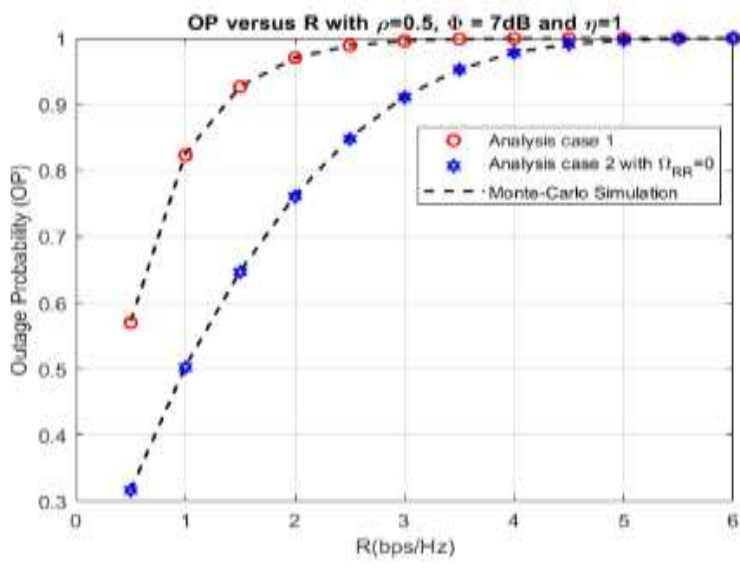

Figure 6. OP versus R

\section{CONCLUSION}

The main idea of this paper is investigated the system performance of energy harvesting FD relaying network over block rayleigh fading channel under the influence of the loopback interference channel. In the first stage, we proposed the system model and analyzed the energy harvesting and the information transmission phases. Furthermore, the mathematical form for the outage probability (OP) is analyzed and derived in two kinds of loopback interference: residual self-interference is modeled as AWGN và residual self-interference is still a random variable. All the mathematical, analytical expressions are verified using the Monte Carlo simulation.

\section{ACKNOWLEDGEMENTS}

This research was supported by the Industrial University of Ho Chi Minh City (IUH), Vietnam, under grantNo. 72/HD-DHCN.

\section{REFERENCES}

[1] Chen, He, Chao Zhai, Yonghui Li, and Branka Vucetic, "Cooperative strategies for wireless-powered communications: an overview," IEEE Wireless Communications, vol. 25, no. 4, pp. 112-119, 2018, doi: 10.1109/mwc.2017.1700245.

[2] Bi, S., Ho, C. K., and Zhang, R., "Wireless powered communication: opportunities and challenges," IEEE Communications Magazine, vol. 53, no. 4, pp. 117-125, 2015, doi: 10.1109/MCOM.2015.7081084.

[3] Niyato, D., Kim, D. I., Maso, M., and Han, Z., "Wireless powered communication networks: research directions and technological approaches," IEEE Wireless Communications, vol. 24, no. 6, 2017, pp. 88-97, 2017, doi: 10.1109/MWC.2017.1600116.

[4] Yu, H., Lee, H., and Jeon, H., "What is 5G? emerging 5G mobile services and network requirements," vol. 9, no. 10, 2017, doi: 10.3390/su9101848.

[5] C. R. Valenta and G. D. Durgin, "Harvesting wireless power: survey of energy-harvester conversion efficiency in far-field, wireless power transfer systems," IEEE Microw. Mag., vol. 15, no. 4, pp. 108-120, Jun. 2014, doi: 10.1109/MMM.2014.2309499.

[6] Sharma, V., and Karmakar, P., "A novel method of opportunistic wireless energy harvesting in cognitive radio networks," 2015 7th International Conference on Computational Intelligence, Communication Systems and Networks, 2015, doi: 10.1109/CICSyN.2015.21. 
[7] A. M. Fouladgar and O. Simeone, "On the transfer of information and energy in multi-user systems," IEEE Communications Letters, vol. 16, no. 11, pp. 1733-1736, 2012, doi: 10.1109/LCOMM.2012.091212.121660.

[8] Zhang, R., and Ho, C. K., "MIMO broadcasting for simultaneous wireless information and power transfer," IEEE Transactions on Wireless Communications, vol. 12, no. 5, pp. 1989-2001, doi:10.1109/twc.2013.031813.120224.

[9] Park, J., and Clerckx, B., "Joint wireless information and energy transfer in a two-user MIMO interference channel," IEEE Transactions on Wireless Communications, vol. 12, no. 8, pp. 4210-4221, 2013, doi: 10.1109/twc.2013.071913.130084.

[10] Chalise, B. K., Zhang, Y. D., and Amin, M. G., "Energy harvesting in an OSTBC based amplify-and-forward MIMO relay system," 2012 IEEE International Conference on Acoustics, Speech and Signal Processing (ICASSP), 2012, doi: 10.1109/icassp.2012.6288596.

[11] Tutuncuoglu, K., and Yener, A., "Cooperative energy harvesting communications with relaying and energy sharing," 2013 IEEE Information Theory Workshop (ITW), 2013, doi: 10.1109/itw.2013.6691280.

[12] Wyner, A. D, "The wire-tap channel," The Bell System Technical Journal, vol. 54, no. 8, pp. 1355-1387, Oct 1975, doi: 10.1002/j.1538-7305.1975.tb02040.x.

[13] Wang, L., Wong, K., Jin, S., Zheng, and G., Jr., R.W.H., "A new look at physical layer security, caching, and wireless energy harvesting for heterogeneous ultra-dense networks," IEEE Communications Magazine, vol. 56, no. 6, pp. 49-55, 2018, doi: 10.1109/MCOM.2018.1700439.

[14] Obeed, M., and Mesbah, W., "Efficient algorithms for physical layer security in two-way relay systems," Physical Communication, vol. 28, pp. 78-88, 2018, doi: 10.1016/j.phycom.2018.03.007.

[15] Shah, H.A., and Koo, I., "A novel physical layer security scheme in ofdm-based cognitive radio networks," IEEE Access, vol. 6, pp. 29486-29498, 2018, doi: 10.1109/ACCESS.2018.2842826.

[16] Tan N. Nguyen, Tran Trung Duy, Gia-Thien Luu, Phuong T. Tran and Miroslav Voznak, "Energy harvesting-based spectrum access with incremental cooperation, relay selection and hardware noises," Radioengineering, vol. 26, no. 1, pp. 240-250, Apr. 2017, doi: 10.13164/re.2017.0240.

[17] Daniel Zwillinger. Table of Integrals, Series, and Products, Academic Press 2015.

[18] J. Mo, M. Tao, and Y. Liu, "Relay placement for physical layer security: a secure connection perspective," IEEE Commun. Lett. vol. 16, no. 6, pp. 878-881, 2012, doi: 10.1109/LCOMM.2012.042312.120582.

[19] T. T. Duy and P. N. Son, "Secrecy performances of multicast underlay cognitive protocols with partial relay selection and without eavesdropper's information," KSII Trans on Internet Inform. Syst., vol. 9, no. 11, pp. 46234643, 2015, doi: :10.3837/tiis.2015.11.021.

[20] S. Jia, J. Zhang, H. Zhao, anf R. Zhang, "Relay selection for improved security in cognitive relay networks with jamming," IEEE Wireless Commun. Lett., vol. 6, no. 5, pp. 662-665, 2017, doi: 10.1109/LWC.2017.2728528.

[21] Nguyen, Tan N, Phu Tran Tin, Phuong T. Tran, Tran Hoang Quang Minh, and Miroslav Voznak, "Power-splitting protocol in power beacon-assisted energy harvesting full-duplex relaying networks: performance analysis," 2018 11th IFIP Wireless and Mobile Networking Conference (WMNC), 09 2018. doi: 10.23919/wmnc.2018.8480900.

[22] Tran Hoang Quang Minh, "Hybrid time-power switching protocol of energy harvesting bidirectional relaying network: throughput and ergodic capacity analysis," TELKOMNIKA (Telecommunication, Computing, Electronics and Control), vol. 16, no. 5, pp. 1947-1956, 2018, doi: 10.12928/telkomnika.v16i5.9118.

[23] Tin, Phu Tran, Tran Hoang Quang Minh, Tan N. Nguyen, and Miroslav Voznak, "System performance analysis of half-duplex relay network over rician fading channel," TELKOMNIKA (Telecommunication, Computing, Electronics and Control), vol. 16, no. 1, pp. 1947-1956, 2018, doi: 10.12928/telkomnika.v16i1.7491.

[24] Rashid, Tarique, Sunil Kumar, Akshay Verma, Prateek Raj Gautam, and Arvind Kumar, "Pm-EEMRP: postural movement based energy efficient multi-hop routing protocol for intra wireless body sensor network (intra-WBSN)," TELKOMNIKA (Telecommunication, Computing, Electronics and Control), vol. 16, no. 1, pp. 166-173, 2018, doi: 10.12928/telkomnika.v16i1.7318.

[25] Tin, Phu Tran, Minh Tran, Tan N. Nguyen, and Thanh-Long Nguyen, "System performance analysis of hybrid time-power switching protocol of EH bidirectional relaying network in amplify-and-forward mode," Indonesian Journal of Electrical Engineering and Computer Science (IJEECS), vol. 14, no. 1, pp. 118-126, 2019, doi: 10.11591/ijeecs.v14.i1.pp118-126. 